\title{
Innovative transcatheter procedures for the treatment of heart failure
}

\author{
Francesco Saia ${ }^{1}$, Antonino Loforte ${ }^{2}$, Davide Pacini ${ }^{2}$ \\ ${ }^{1}$ Cardiology Unit, Cardio-Thoracic-Vascular Department, ${ }^{2}$ Cardiac Surgery Unit, Cardio-Thoracic-Vascular Department, University Hospital of \\ Bologna, Policlinico S. Orsola-Malpighi, Bologna, Italy \\ Contributions: (I) Conception and design: F Saia, A Loforte; (II) Administrative support: None; (III) Provision of study materials or patients: F Saia, \\ D Pacini; (IV) Collection and assembly of data: None; (V) Data analysis and interpretation: None; (VI) Manuscript writing: All authors; (VII) Final \\ approval of manuscript: All authors. \\ Correspondence to: Francesco Saia, MD, PhD. Cardiology Unit, Cardio-Thoracic-Vascular Department, University Hospital of Bologna, Policlinico \\ S.Orsola-Malpighi (Pav. 23), Via Massarenti, 9, 40138 Bologna, Italy. Email: francescosaia@hotmail.com.
}

\begin{abstract}
The prevalence of heart failure (HF) continues to rise over time, with aging of the population and increased survival of incident cases. Major improvements occurred in drug therapy but morbidity and mortality of HF patients remain high. Some non-pharmacologic approaches to HF are already part of standard treatment for HF, including implantable cardioverter-defibrillators, cardiac resynchronization therapy (CRT) and left ventricular assist devices (LVADs). A number of transcatheter treatments and devices have been developed to improve management of valvular heart diseases (VHD), and some of them are being used or tested in specific HF conditions. For example, transcatheter aortic valve implantation (TAVI) to unload the left ventricle in patients with moderate aortic stenosis (AS) and HF or TAVI for severe aortic regurgitation (AR) in patients with LVADs. Similarly, percutaneous mitral valve repair can be used to improve prognosis and quality of life in patients with functional mitral valve regurgitation, and has been proposed as a bridge-to-LVAD or to heart transplant in selected patients. Other devices have been specifically developed for the treatment of chronic HF. In this review we describe the main devices used in the treatment of $\mathrm{HF}$ associated with aortic and mitral valve disease, as well as novel transcatheter interventions for chronic HF with different pathophysiologic targets.
\end{abstract}

Keywords: Heart failure (HF); transcatheter aortic valve replacement; percutaneous mitral valve repair; left ventricular dysfunction; secondary mitral regurgitation; left ventricular assist device (LVAD)

Submitted Mar 09, 2020. Accepted for publication Apr 22, 2020.

doi: $10.21037 / \mathrm{cdt}-20-335$

View this article at: http://dx.doi.org/10.21037/cdt-20-335

\section{Introduction}

The prevalence of heart failure (HF) continues to rise over time, with aging of the population and increased survival of incident cases (1). HF is a debilitating disease that decreases life expectancy and quality of life and is responsible for a large number of hospital admission and healthcare resource consumption. Virtually all cardiovascular diseases may lead to HF, although prevalence, causes and risk factors of HF may vary worldwide. In some cases, HF can be treated by eliminating its primary cause (e.g., valvular heart disease, VHD), in others however, pharmacologic and nonpharmacologic treatments are aimed at reducing rate of progression and mortality related to $\mathrm{HF}$ and to improve quality of life (e.g., cardiomyopathies).

Several non-pharmacologic strategies for the treatment of HF have been already tested and represent standard treatments for HF in selected patients at different stages, including cardiac resynchronization therapy (CRT) and left ventricular assist devices (LVADs) (2). In recent years, development of novel transcatheter techniques, especially for the treatment of VHD, which may cause or aggravate 


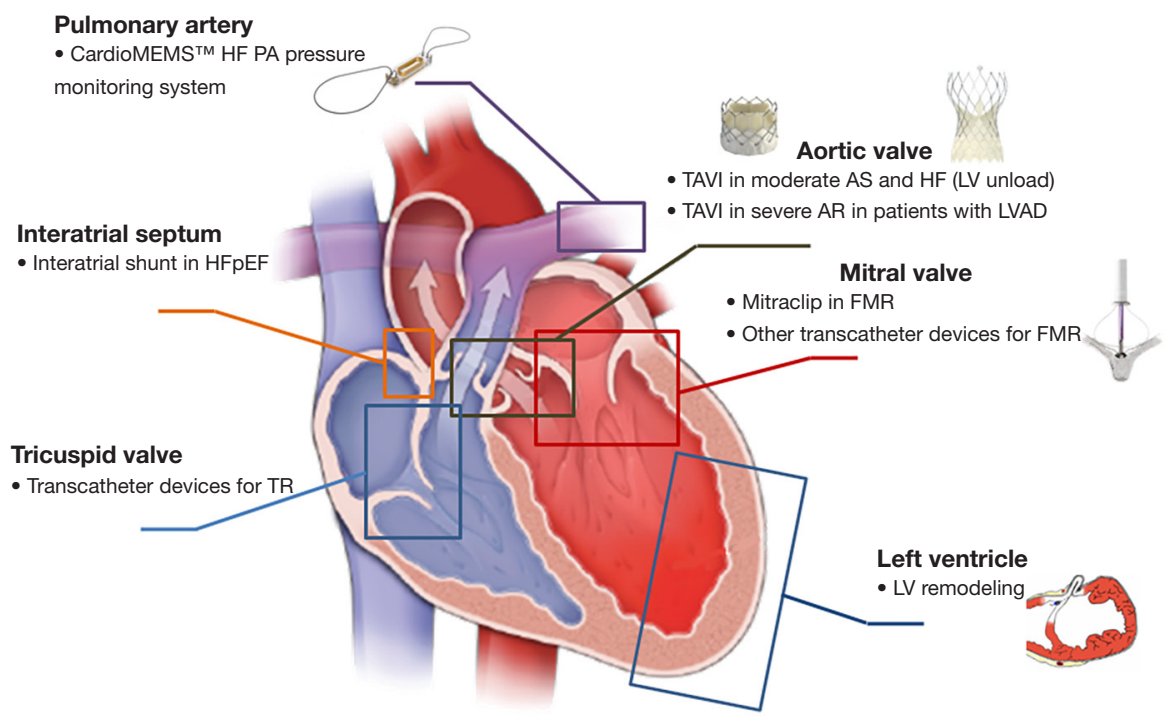

Figure 1 Targets of new transcatheter options for patients with heart failure. AS, aortic stenosis; FMR, functional mitral regurgitation; HF, heart failure; HFpEF, heart failure with preserved ejection fraction; LV, left ventricle; PA, pulmonary artery; TAVI, transcatheter aortic valve implantation; TR, tricuspid regurgitation.

HF, offered new treatment options for patients with HF (Figure 1). This paper provides a brief review of the current applications of new transcatheter techniques for HF patients.

\section{Transcatheter aortic valve implantation (TAVI) in HF}

TAVI has become a mainstay therapy for elderly patients with severe aortic stenosis (AS) with increased surgical risk $(3,4)$, and recently published trials will probably lead to expanded indications to low-risk patients as well $(5,6)$. As such, TAVI already represents an extremely valuable therapy for HF due to severe AS. Beyond common indications, however, TAVI is under investigations in other specific HF scenarios.

The Transcatheter Aortic Valve Replacement to UNload the Left ventricle in patients with ADvanced heart failure (TAVR UNLOAD, NCT02661451) is an international, multicenter, randomized, open-label, clinical trial designed to test the efficacy and the safety of transfemoral TAVI in HF patients with reduced left ventricular ejection fraction (LVEF) and moderate AS (7). In TAVR-UNLOAD, 600 patients with $\mathrm{HF}$ and $\mathrm{LVEF}<50 \%$, NYHA $\geq 2$, optimal HF therapy (OHFT) and moderate AS are being randomized 1:1 to undergo TAVR+OHFT $v$ s. OHFT alone. The primary endpoint will be the hierarchical occurrence at 12-month of: all-cause death, disabling stroke, hospitalizations for HF, and change in KCCQ. The rationale of the study is that augmented systemic vascular resistance due to moderate AS may contribute to worsening of myocardial function and symptoms. Indeed, decreasing vascular resistance is one of the main targets of pharmacologic treatment of HF. A case report from Bastos et al. provide proof of the concept tested in the trial (8). In fact, immediately after TAVI in a patient with $\mathrm{HF}$ and moderate AS, important hemodynamic changes were recorded, including left shifting of the pressurevolume loops with decreases in end-diastolic and endsystolic volumes, increase in end-systolic elastance indicating improved contractility, decreased pressure-volume area, lower ventricular-arterial coupling ratio and increase of LVEF (8).

TAVI has also been proposed in other, very specific end-stage HF settings: as a bridge to heart transplant or to LVAD in patients with end-stage HF and severe native or bioprosthetic aortic valve dysfunction (9), or to treat acquired aortic insufficiency after LVAD implantation $(10,11)$. Severe aortic regurgitation (AR) is a well-known potential complication of LVAD, whose utilization has greatly increased over the last decade. AR has been mainly reported after continuous-flow LVAD, with $\geq$ moderate AR occurring in nearly a third of the patients after 3 years of continuous-flow LVAD support, and often leads to recurrent $\mathrm{HF}$ and reduced survival $(11,12)$. In these patients, surgical treatment of AR is complex and poses 
a high risk; hence, a less invasive, transcatheter option like TAVI has been explored $(11,12)$. Several cases have been reported, with overall good results (12). Likewise, a successful TAVI case was also reported in a patient with pulsatile-flow biventricular assist device (10). The principal limitation of TAVI in this setting is the lack of valve calcifications in the majority of cases, with the consequent risk of valve embolization and malpositioning. For the most commonly used TAVI devices, treatment of pure AR is offlabel (13). An alternative transcatheter option is application of a septal occluder device to close the aortic valve and avoid regurgitation of a portion of the forward flow from the LVAD outflow cannula into the left ventricle. Although reported to be effective, a potential drawback of this approach is that the patient becomes fully dependent on the LVAD and its malfunction can quickly become fatal (11).

\section{Transcatheter mitral valve interventions in HF}

Mitral valve regurgitation (MR) can be either the cause (primary or organic MR) or a consequence (secondary or functional MR) of left ventricular dysfunction and HF. For primary MR, surgery, preferably repair over replacement, is strongly indicated in most patients (class I or IIa), with a lower degree of recommendation for mitral valve replacement in symptomatic patients with severe $\mathrm{LV}$ dysfunction (LVEF $<30 \%$ and/or LVESD >55 mm) who are refractory to medical therapy (3). Nevertheless, a sizable number of patients cannot undergo surgery because of advanced age and/or comorbidity, and this proportion is increasing due to aging population. To cover this unmet need, several transcatheter approaches have been developed and are at different stages of clinical or pre-clinical testing. The MV apparatus is more complex than the aortic valve and targets for interventions might be manifold: leaflets, chordae, and annulus. Accordingly, there are devices designed for transcatheter edge-to-edge procedures, annuloplasty (direct or indirect), mitral chord replacement, or transcatheter mitral valve replacement (TMVR). For secondary MR, surgical indications are much less straightforward, because the primary disease involves the left ventricle and surgical correction of MR is burdened with high surgical risk and uncertain prognostic impact. As a consequence, the vast majority of these patients are managed conservatively.

The most used device for transcatheter mitral valve repair is the MitraClip (Abbott, Abbott Park, IL, USA), which simulates the Alfieri surgical technique in a percutaneous fashion. MitraClip has been approved to treat symptomatic but inoperable patients with primary MR if the anatomy allows (14). Once again, the situation is much more complex for secondary MR. Two randomized trials evaluating MitraClip vs. medical treatment in functional MR provided apparently contradicting results. In the Multicentre Study of Percutaneous Mitral Valve Repair with MitraClip Device in Patients With Severe Secondary Mitral Regurgitation (MITRA-FR) (15) the composite primary endpoint for allcause mortality and HF hospitalization at 1-year was not significantly different between treatment groups, and this lack of difference was confirmed in a 2-year follow-up (16). Conversely, in the Cardiovascular Outcomes Assessment of the MitraClip Percutaneous Therapy for Heart Failure Patients with Functional Mitral Regurgitation (COAPT) trial, MitraClip implantation led to a significant reduction in the primary endpoint of HF hospitalization at 2-year follow-up versus medical treatment alone. Surprisingly, MitraClip was also associated with significantly lower mortality and better quality of life. Correlating the findings of those 2 studies represents a pathophysiologic challenge with huge clinical implications, given the enormous potential number of candidates with HF and secondary MR.

First of all, both trials confirmed the ominous fate of medically-treated patients. Second, patients enrolled in the MITRA-FR trial had on average less severe MR and greater LV dilatation. Echocardiographic criteria for enrollment were less stringent than in COAPT (17), and adjustments of medical treatment during the study were more frequent, whereas in COAPT guideline-directed medical treatment had to be fully implemented at the time of enrollment in the trial. Based on the analysis of those trials, a novel conceptual framework has been proposed to guide therapeutic approach (18). Briefly, in secondary MR, the left ventricle has a critical importance and effective regurgitant orifice area (EROA) is dependent on left ventricular enddiastolic volume. A certain degree of MR should therefore be expected with a direct proportionality with LV dilatation: this has been named "proportionate" secondary MR. In this context treating the mitral valve is supposed to have minor or null impact on outcomes. Some patients, however, presents with an EROA and a degree of MR that is disproportionately higher than predicted by LV dilatation. This condition has been named "disproportionate" or "tertiary" MR and is the one that appears to derive more benefit from interventions directed at the mitral valve (18). This theory is, however, still subject to different interpretations (19). Overall, it seems very rationale to 
Table 1 Potential indications to percutaneous mitral valve repair (PMVR) as a bridge to heart transplant*

\begin{tabular}{|c|c|c|c|}
\hline Status & Condition & Potential indication to PMVR & Therapeutic objective \\
\hline \multirow{2}{*}{ Active HTx list } & Unstable hemodynamics & Relevant contribution of FMR to instability & Avoid deterioration while on waiting list \\
\hline & Rare blood group & Unstable condition & Avoid deterioration while on waiting list \\
\hline \multirow[t]{3}{*}{$\begin{array}{l}\text { Potential } \\
\text { candidate to HTx }\end{array}$} & $\begin{array}{l}\text { Non-cardiac temporary } \\
\text { contraindication }\end{array}$ & Unstable condition & Avoid deterioration while on waiting list \\
\hline & Pulmonary hypertension & PVR $>3.0 \mathrm{WU}$ or $\mathrm{PCW}>30 \mathrm{mmHg}$ & Reduction of PVR \\
\hline & Unstable hemodynamics & Relevant contribution of FMR to instability & $\begin{array}{l}\text { Improve symptoms and hemodynamic } \\
\text { conditions during the pre-transplant } \\
\text { screening phase }\end{array}$ \\
\hline
\end{tabular}

*, reproduced from Saia et al. (23) with permission. FMR, functional mitral regurgitation; HTx, heart transplant; PAP, pulmonary arterial pressure; PCW, pulmonary capillary wedge; PVR, pulmonary vascular resistance; RHC, right heart catheterization; WU, Woods units.

preferentially apply MitraClip therapy according to the enrollment criteria of the COAPT trial (17). While it should be tried to avoid futile procedures, it is clear that patients with $\mathrm{HF}$ and functional MR represent a complex scenario and a holistic approach is necessary for each individual patient. In fact, some registry data suggest clinical benefit even in some patients with truly end-stage HF (20), despite the lack of impact on parameters strictly linked to survival (e.g., left ventricular remodeling). It is difficult, however, to match these findings from those, more rigorous that have emerged from randomized trials. A third ongoing clinical trial, the RESHAPEHF2 (NCT02444338), will provide further evidence and hopefully help refine appropriate patient selection for MitraClip in secondary MR.

A niche indication for MitraClip that has been recently proposed by some heart transplant centers is bridge to heart transplant or to LVAD in selected candidates $(21,22)$ (Table 1). Indications include waitlisted patients with rapidly-deteriorating clinical condition and initial loss of end-organ functions, patients with potentially-reversible contraindications to heart transplant (i.e., pulmonary hypertension) or unstable patients during the screening for heart transplant (before entering the waiting list) (23).

\section{Left ventricular remodeling}

Scar formation after myocardial infarction results in progressive $\mathrm{LV}$ remodeling that, in some instances, can lead to formation of an aneurysm. LV aneurysm increases wall tension and reduces the efficacy of $\mathrm{LV}$ contractions, leading to $\mathrm{HF}$ and increasing risk of endoventricular thrombus formation. Hence, cardiac surgeons developed a technique to resect the $\mathrm{LV}$ aneurysm, regain a more physiological $\mathrm{LV}$ shape, improve LV function and reduce the risks associated with the presence of the aneurysm. Although in the Surgical Treatment for Ischemic Heart Failure (STICH) randomized clinical trial no difference was demonstrated for the primary outcome of death from any cause or rehospitalization for HF (24), a subsequent analysis suggested a potential survival after CABG and $\mathrm{LV}$ reconstruction compared to CABG alone when a postoperative end-systolic volume index of $70 \mathrm{~mL} / \mathrm{m}$ or less was achieved (25). Given these results and the high surgical risk, LV aneurysmectomy is performed only in very selected patients.

Recently, a new transcatheter approach for ventricular reshaping have been developed: the Revivent $\mathrm{TC}^{\mathrm{TM}}$ System (BioVentrix Inc., San Ramon, CA, USA) (26). Revivent is a hybrid transcatheter/surgical technique in which the $\mathrm{LV}$ is reconstructed by plication of the fibrous scar on a beating heart. A series of titanium microanchors are implanted internally from the right ventricle cavity through a jugular venous access and externally from the LV free wall through a surgical access, and connected by an adjustable-length tether (Figure 2). A series of anchors are implanted until plication of the aneurysm is obtained. Preliminary data showed significant and sustained reduction of $L V$ volumes and improvement of LV function, symptoms, and quality of 
A

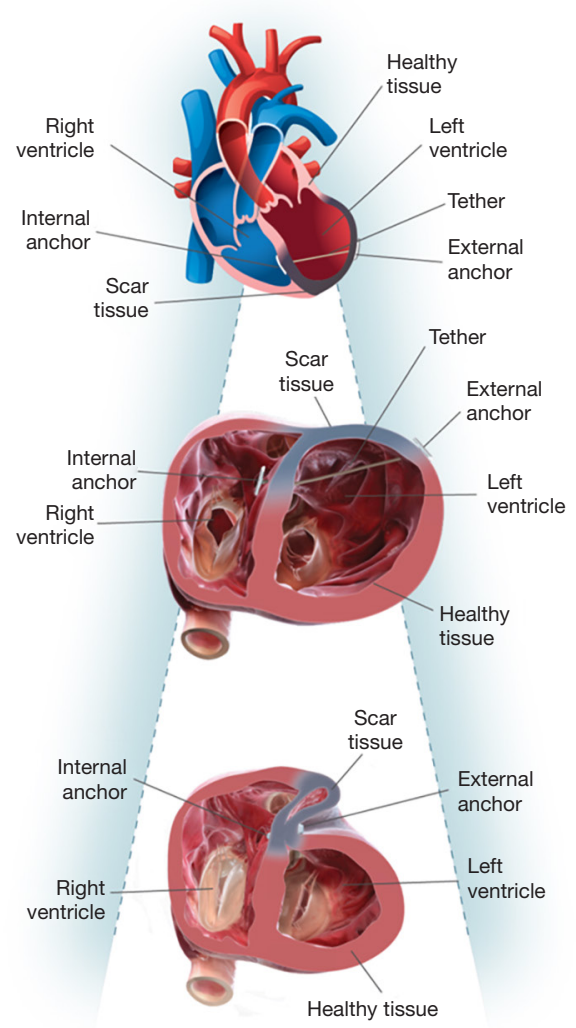

B
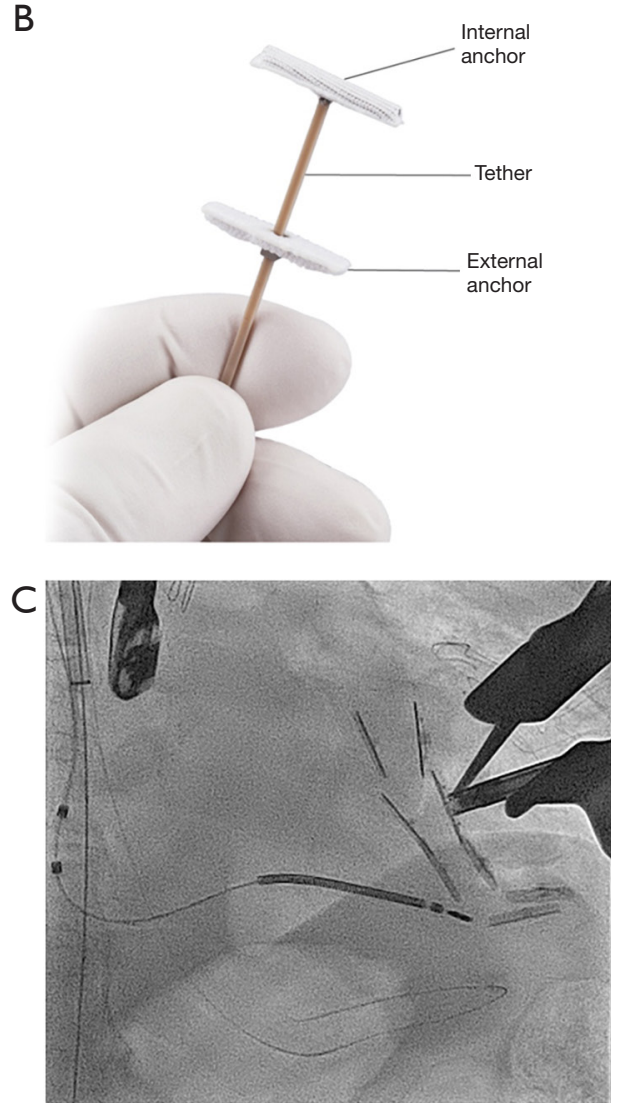

Figure 2 The Revivent TC ${ }^{\mathrm{TM}}$ device. (A)* Illustration of the procedure. (B)* Focus on the nitinol anchors for plication of the left ventricular aneurism. (C) Intra-operative fluoroscopic image. *, from https://bioventrix.com/index.php/it/medico/sistema-revivent-tc.

life $(27,28)$. Further studies are ongoing.

\section{Remote monitoring of HF signals}

Remote monitoring of symptoms or physiologic signals have been proposed as possible strategies to reduce hospitalizations for HF, through the detection of early signs of cardiac decompensation and prompt initiation of the appropriate treatment before development of acute HF. Several attempts have been made with inconsistent results, including: daily weight measurements, patient reported symptoms, B-type natriuretic peptide levels, or nonhaemodynamic physiologic signals derived from implanted devices, such as intrathoracic impedance $(29,30)$.

Increased intravascular volume is often associated with elevation of pulmonary artery (PA) pressure that might be apparent several days before the onset of worsening symptoms. The CardioMEMS ${ }^{\mathrm{TM}}$ HF System (Abbott, Atlanta, GA, USA) is a wireless implantable haemodynamic monitoring system implantable into a distal branch of the PA which enables transmission of pressure readings and trends to HF clinics (Figure 1). In the CHAMPION prospective, randomized clinical trial (ClinicalTrials.gov NCT00531661) medical management guided by the CardioMEMS ${ }^{\mathrm{TM}}$ through remotely obtained PA pressures has been shown to significantly reduce PA pressure, HF hospitalizations and improve quality of life in patients with NYHA Class III HF (31). After CE mark and FDA approval, the device is being investigated in a European post-market study designed to test generalizability and efficacy of remote hemodynamic guided HF management (30).

\section{Catheter-based techniques for cardiac support in acute HF}

Acute decompensated heart failure (ADHF) is an unstable condition characterized by new onset or worsening HF symptoms, that in some cases may present or evolve to 
cardiogenic shock. If patients do not respond adequately to volume optimization and inotropes, mechanical circulatory support (MCS) is recommended to unload the ventricle and maintain sufficient end-organ perfusion (2). Devices for temporary MCS can be either percutaneous or surgical. Along with the traditional intra-aortic balloon pump (IABP), the most important catheter-based devices include the Impella (Abiomed, Danvers, MA, USA) and the TandemHeart (Cardiac Assist Inc, Pittsburgh, PA, USA).

Impella is a micro-axial pump which is placed in the $\mathrm{LV}$ and delivers blood through the LV to the ascending aorta. It acts as a percutaneous LVAD, generating an effective $\mathrm{LV}$ unloading and decreasing myocardial oxygen. TandemHeart consists of a transseptal cannula inserted from the femoral vein that aspires blood in the left atrium and, through a centrifugal blood pump, reintroduce it into the femoral artery. They provide superior hemodynamic support compared with IABP but are also associated with higher rates of complications, hence selective use within a welldefined therapeutic protocol is recommended (32).

\section{Percutaneous revascularization for ischemic ventricular dysfunction}

Coronary artery disease is the most common cause of left ventricular dysfunction and HF. In these patients, revascularization with coronary artery bypass graft (CABG) improves long-term outcomes, despite a high surgical risk (33). The role of percutaneous coronary intervention (PCI) is less clear and indirect comparisons suggest superiority of CABG (34). Despite the lack of randomized data, however, PCI seems superior to medical therapy alone (34) and clinical trials are ongoing (35). Many patients have comorbidities that further increase an already-high surgical risk, making PCI the best therapeutic strategy. Yet, in this setting PCI is burdened by important drawbacks. The ability to achieve more often a complete revascularization is one of the possible explanations for the hypothesized superiority of CABG over PCI (35). Second, complex PCI procedures are often required in this patients' cohort, carrying a high procedural risk. Importantly, a number of innovations for PCI have been recently introduced in clinical practice that might increase safety and effectiveness in patients with severe $L V$ dysfunction. Amelioration of materials, techniques, success rates and clinical outcomes in chronic total occlusions (CTO) PCI have been reported (36). New-generation MCS devices allow safer performance of complex procedures in patients with severe LV dysfunction and hemodynamic instability, complex multivessel disease or last-remaining vessel $(37,38)$.

\section{Other devices}

A number of other devices for the treatment of HF have been developed and are being tested in clinical trials. First of all, a large number of new emerging technologies are under development to treat mitral regurgitation (39) and tricuspid regurgitation (40). The Parachute device (CardioKinetix; Menlo Park, CA, USA) was aimed at treating $\mathrm{LV}$ aneurysms by partitioning apical $\mathrm{LV}$ aneurysms through a self-expanding endoventricular nitinol device (26). Testing of the Parachute has been abandoned because of the closure of the company. Another interesting concept is that of creating an interatrial shunt in patients with $\mathrm{HF}$ with preserved LVEF (HFpHF) to reduce left atrial pressure. In these patients, baseline left atrial pressure is high, and there is a steep increase during exercise, causing rise in pulmonary capillary wedge pressure (PCWP) and exertional dyspnea. The transcatheter Interatrial Shunt Device (IASD; Corvia Medical Inc.) has been shown to be safe and associated with a reduction of exercise PCWP compared with sham control treatment at 1 month of follow-up, but at 1 -year there were no significant differences in the combined endpoint of major adverse cardiac, cerebrovascular, or renal events (41). However, the trial was underpowered to detect significant differences in 12-month end points and further studies are ongoing. Another device, the $\mathrm{V}$-wave device ( $\mathrm{V}$-Wave Medical), which is an hourglass-shaped interatrial shunt device that includes a valve, had a rate of device malfunction or failure of $50 \%$ at 1-year (41).

\section{Conclusions}

$\mathrm{HF}$ is a major public health problem and has increasing prevalence. Guideline-directed medical therapy is associated with improved outcomes and quality of life, but many patients remain symptomatic and prognosis is often dismal. A number of non-pharmacologic approaches complement the therapeutic armamentarium for HF. Several transcatheter devices are now available in clinical practice or are under evaluation for HF patients, and will help to improve prognosis and quality of life.

\section{Acknowledgments}

This paper was presented in the joint session of the 27 th 
Annual Meeting of the ISMCS 2019 and RHICS 17th Expert Forum, October 21-23, 2019, Bologna, Italy. Funding: None.

\section{Footnote}

Provenance and Peer Review: This article was commissioned by the Guest Editor (Roland Hetzer) for the series "Heart Failure in the Young and Old: Insights into Various Therapies" published in Cardiovascular Diagnosis and Therapy. The article has undergone external peer review.

Conflicts of Interest: All authors have completed the ICMJE uniform disclosure forms (available at http://dx.doi. org/10.21037/cdt-20-335). The series "Heart Failure in the Young and Old: Insights into Various Therapies" was commissioned by the editorial office without any funding or sponsorship. FS reports personal fees from Abbott Vascular, personal fees from Edwards, personal fees from Medtronic, personal fees from Boston Scientific, personal fees from Astra Zeneca, personal fees from Daiichy-Sankyo, personal fees from Amgen, personal fees from Bayer, personal fees from Boheringer-Ingelheim, outside the submitted work. The authors have no other conflicts of interest to declare.

Ethical Statement: The authors are accountable for all aspects of the work in ensuring that questions related to the accuracy or integrity of any part of the work are appropriately investigated and resolved.

Open Access Statement: This is an Open Access article distributed in accordance with the Creative Commons Attribution-NonCommercial-NoDerivs 4.0 International License (CC BY-NC-ND 4.0), which permits the noncommercial replication and distribution of the article with the strict proviso that no changes or edits are made and the original work is properly cited (including links to both the formal publication through the relevant DOI and the license). See: https://creativecommons.org/licenses/by-nc-nd/4.0/.

\section{References}

1. Virani SS, Alonso A, Benjamin EJ, et al. Heart Disease and Stroke Statistics-2020 Update: A Report From the American Heart Association. Circulation 2020;141:e139-e596.

2. Ponikowski P, Voors AA, Anker SD, et al. 2016 ESC Guidelines for the diagnosis and treatment of acute and chronic heart failure: The Task Force for the diagnosis and treatment of acute and chronic heart failure of the European Society of Cardiology (ESC). Developed with the special contribution of the Heart Failure Association (HFA) of the ESC. Eur J Heart Fail 2016;18:891-975.

3. Baumgartner H, Falk V, Bax JJ, et al. 2017 ESC/EACTS Guidelines for the management of valvular heart disease. Eur Heart J 2017;38:2739-91.

4. Nishimura RA, Otto CM, Bonow RO, et al. 2017 AHA/ ACC Focused Update of the 2014 AHA/ACC Guideline for the Management of Patients With Valvular Heart Disease: A Report of the American College of Cardiology/ American Heart Association Task Force on Clinical Practice Guidelines. J Am Coll Cardiol 2017;70:252-89.

5. Mack MJ, Leon MB, Thourani VH, et al. Transcatheter Aortic-Valve Replacement with a Balloon-Expandable Valve in Low-Risk Patients. N Engl J Med 2019;380:1695-705.

6. Popma JJ, Deeb GM, Yakubov SJ, et al. Transcatheter Aortic-Valve Replacement with a Self-Expanding Valve in Low-Risk Patients. N Engl J Med 2019;380:1706-15.

7. Spitzer E, Van Mieghem NM, Pibarot P, et al. Rationale and design of the Transcatheter Aortic Valve Replacement to UNload the Left ventricle in patients with ADvanced heart failure (TAVR UNLOAD) trial. Am Heart J 2016;182:80-8.

8. B Bastos M, Schreuder JJ, Daemen J, et al. Hemodynamic Effects of Transcatheter Aortic Valve Replacement for Moderate Aortic Stenosis With Reduced Left Ventricular Ejection Fraction. JACC Cardiovasc Interv 2019;12:684-6.

9. Pascual I, Diaz-Molina B, Lambert JL, et al. "Valve in valve" through femoral approach to allow the later implantation of long-term left ventricle assist device: a case report. J Thorac Dis 2018;10:E447-9.

10. Gomes B, Bekeredjian R, Leuschner F, et al. Transfemoral aortic valve replacement for severe aortic valve regurgitation in a patient with a pulsatile-flow biventricular assist device. ESC Heart Fail 2019;6:217-21.

11. Kalathiya RJ, Grinstein J, Uriel N, et al. Percutaneous Transcatheter Therapies for the Management of Left Ventricular Assist Device Complications. J Invasive Cardiol 2017;29:151-62.

12. Yehya A, Rajagopal V, Meduri C, et al. Short-term results with transcatheter aortic valve replacement for treatment of left ventricular assist device patients with symptomatic aortic insufficiency. J Heart Lung Transplant 2019;38:920-6.

13. Wernly B, Eder S, Navarese EP, et al. Transcatheter aortic 
valve replacement for pure aortic valve regurgitation: "onlabel" versus "off-label" use of TAVR devices. Clin Res Cardiol2019;108:921-30.

14. Feldman T, Foster E, Glower DD, et al. Percutaneous repair or surgery for mitral regurgitation. $\mathrm{N}$ Engl J Med 2011;364:1395-406.

15. Obadia JF, Messika-Zeitoun D, Leurent G, et al. Percutaneous Repair or Medical Treatment for Secondary Mitral Regurgitation. N Engl J Med 2018;379:2297-306.

16. Iung B, Armoiry X, Vahanian A, et al. Percutaneous repair or medical treatment for secondary mitral regurgitation: outcomes at 2 years. Eur J Heart Fail 2019;21:1619-27.

17. Asch FM, Grayburn PA, Siegel RJ, et al. Echocardiographic Outcomes After Transcatheter Leaflet Approximation in Patients With Secondary Mitral Regurgitation: The COAPT Trial. J Am Coll Cardiol 2019;74:2969-79.

18. Grayburn PA, Sannino A, Packer M. Proportionate and Disproportionate Functional Mitral Regurgitation: A New Conceptual Framework That Reconciles the Results of the MITRA-FR and COAPT Trials. JACC Cardiovasc Imaging 2019;12:353-62.

19. Gaasch WH, Aurigemma GP, Meyer TE. An Appraisal of the Association of Clinical Outcomes With the Severity of Regurgitant Volume Relative to End-Diastolic Volume in Patients With Secondary Mitral Regurgitation. JAMA Cardiol 2020;5:476-81.

20. Berardini A, Biagini E, Saia F, et al. Percutaneous mitral valve repair: The last chance for symptoms improvement in advanced refractory chronic heart failure? Int J Cardiol 2017;228:191-7.

21. Geis NA, Pleger ST, Bekeredjian R, et al. Haemodynamic effects of percutaneous mitral valve edge-to-edge repair in patients with end-stage heart failure awaiting heart transplantation. ESC Heart Fail 2018;5:892-901.

22. Godino C, Scotti A, Agricola E, et al. Young Patient with Advanced Heart Failure No Longer a Candidate for Heart Transplantation after MitraClipA(R) Procedure. J Heart Valve Dis 2017;26:234-6.

23. Saia F, Berardini A, Godino C, et al. Bridge transcatheter mitral valve repair in patient with end-stage heart failure. In: Basso C, Musumeci G, Saia F, et al. editors Percutaneous approaches to mitral valve disease (ISSN 978-88-5532-0030). Minerva Medica, Torino, 2019:131-6.

24. Jones RH, Velazquez EJ, Michler RE, et al. Coronary bypass surgery with or without surgical ventricular reconstruction. N Engl J Med 2009;360:1705-17.

25. Michler RE, Rouleau JL, Al-Khalidi HR, et al. Insights from the STICH trial: change in left ventricular size after coronary artery bypass grafting with and without surgical ventricular reconstruction. J Thorac Cardiovasc Surg 2013;146:1139-45.e6.

26. Nijenhuis VJ, Sanchis L, van der Heyden JAS, et al. The last frontier: transcatheter devices for percutaneous or minimally invasive treatment of chronic heart failure. Neth Heart J 2017;25:536-44.

27. Wang Y, Xiao G, Zhang G, et al. Early Results of the Revivent TC Procedure for Treatment of Left Ventricular Aneurysm and Heart Failure due to Ischemic Cardiomyopathy. EuroIntervention 2020. doi: 10.4244/ EIJ-D-19-00225.

28. Klein P, Anker SD, Wechsler A, et al. Less invasive ventricular reconstruction for ischaemic heart failure. Eur J Heart Fail 2019;21:1638-50.

29. Koehler F, Koehler K, Deckwart O, et al. Efficacy of telemedical interventional management in patients with heart failure (TIM-HF2): a randomised, controlled, parallel-group, unmasked trial. Lancet 2018;392:1047-57.

30. Cowie MR, de Groote P, McKenzie S, et al. Rationale and design of the CardioMEMS Post-Market Multinational Clinical Study: COAST. ESC Heart Fail 2020;7:865-72.

31. Abraham WT, Adamson PB, Bourge RC, et al. Wireless pulmonary artery haemodynamic monitoring in chronic heart failure: a randomised controlled trial. Lancet 2011;377:658-66.

32. Thiele H, Ohman EM, de Waha-Thiele S, et al. Management of cardiogenic shock complicating myocardial infarction: an update 2019. Eur Heart J 2019;40:2671-83.

33. Velazquez EJ, Lee KL, Jones RH, et al. Coronary-Artery Bypass Surgery in Patients with Ischemic Cardiomyopathy. N Engl J Med 2016;374:1511-20.

34. Wolff G, Dimitroulis D, Andreotti F, et al. Survival Benefits of Invasive Versus Conservative Strategies in Heart Failure in Patients With Reduced Ejection Fraction and Coronary Artery Disease: A Meta-Analysis. Circ Heart Fail 2017;10:e003255.

35. Perera D, Clayton T, Petrie MC, et al. Percutaneous Revascularization for Ischemic Ventricular Dysfunction: Rationale and Design of the REVIVED-BCIS2 Trial: Percutaneous Coronary Intervention for Ischemic Cardiomyopathy. JACC Heart Fail 2018;6:517-26.

36. Tajti $\mathrm{P}$, Burke $\mathrm{MN}$, Karmpaliotis D, et al. Update in the Percutaneous Management of Coronary Chronic Total Occlusions. JACC Cardiovasc Interv 2018;11:615-25.

37. O'Neill WW, Kleiman NS, Moses J, et al. A prospective, randomized clinical trial of hemodynamic support with 
Impella 2.5 versus intra-aortic balloon pump in patients undergoing high-risk percutaneous coronary intervention: the PROTECT II study. Circulation 2012;126:1717-27.

38. Ameloot K, B Bastos M, Daemen J, et al. New-generation mechanical circulatory support during high-risk PCI: a cross-sectional analysis. EuroIntervention 2019;15:427-33.

39. Mangieri A, Laricchia A, Giannini F, et al. Emerging Technologies for Percutaneous Mitral Valve Repair. Front Cardiovasc Med 2019;6:161.

40. Overtchouk P, Piazza N, Granada J, et al. Advances

Cite this article as: Saia F, Loforte A, Pacini D. Innovative transcatheter procedures for the treatment of heart failure. Cardiovasc Diagn Ther 2021;11(1):292-300. doi: 10.21037/cdt20-335 in transcatheter mitral and tricuspid therapies. BMC Cardiovasc Disord 2020;20:1.

41. Shah SJ, Feldman T, Ricciardi MJ, et al. One-Year Safety and Clinical Outcomes of a Transcatheter Interatrial Shunt Device for the Treatment of Heart Failure With Preserved Ejection Fraction in the Reduce Elevated Left Atrial Pressure in Patients With Heart Failure (REDUCE LAPHF I) Trial: A Randomized Clinical Trial. JAMA Cardiol 2018;3:968-77. 